\title{
A los niños que tienen convulsiones febriles ¿hay que administrarles anticonvulsivantes cuando tienen fiebre?
}

\author{
M. Aparicio ${ }^{a}$, R. Sánchez-Andrade ${ }^{b}$, MP. González Rodríguez ${ }^{c}$ \\ ${ }^{a}$ Pediatra. CS Entrevías. Servicio Madrileño de Salud, Área I. Madrid. \\ ${ }^{b}$ Pediatra. CS Luis Vives. Servicio Madrileño de Salud, Área 3. Alcalá de Henares, Madrid. \\ 'Pediatra. CS Barrio del Pilar. Servicio Madrileño de Salud, Área 5. Madrid.
}

\begin{abstract}
Resumen
Conclusión de los autores: en el primer ensayo clínico concluyen que el uso intermitente de diazepam rectal $(D R)$ en los procesos febriles, reduce un $45 \%$ el riesgo de recurrencia de convulsiones febriles (CF) en los niños de alto riesgo $(p=0,005)$, un $20 \%$ en los de riesgo intermedio $(p=0,341)$ y un $13 \%$ en los de bajo riesgo $(p=0,412)$. Los efectos secundarios en el grupo con $D R$ fueron moderados y transitorios sin efectos secundarios a largo plazo. La guía de práctica clínica revisa las publicaciones que tratan sobre la prevención de las recurrencias de las CF simples y concluye que aunque hay evidencia de que el tratamiento, ya sea continuado con antiepilépticos o intermitente con diazepam oral (DO), es eficaz para reducir las recurrencias, la posible toxicidad supera los escasos riesgos de las CF. En situaciones de ansiedad familiar, el DO al comienzo de la fiebre puede ser útil para prevenir las recurrencias.

Comentario de los revisores: la profilaxis de las CF con anticonvulsivantes, tanto de forma continua como intermitente, no está indicada de forma general dado que sus riesgos no superan los beneficios del tratamiento. Se podría utilizar en casos particulares de gran angustia familiar ante las recurrencias, beneficiándose más los niños con dos o más factores de riesgo. En estos casos se recomendaría la administración intermitente de $D O$ o $D R$, preferiblemente el segundo, a dosis de $0,33 \mathrm{mg} / \mathrm{kg}$ con un total de dos a tres dosis durante la primeras 24 horas de la fiebre.
\end{abstract}

Palabras clave: Convulsiones febriles, Tratamiento preventivo, Pronóstico.

\section{Abstract}

Authors' conclusion: in the first randomized controlled trial, the intermittent rectal diazepam $(R D)$ prophylaxis reduces $45 \%$ the recurrence rates of febrile seizures in high risk patients $(p=0.005), 20 \%$ reduction in the intermediate-risk $(p=0.341)$ and only $13 \%$ in the low-risk children $(p=0.412)$. The side effects in the RD group were mild and transient and

Este artículo se publica simultáneamente con la revista electrónica Evidencias en Pediatría (EeP, www.aepap.org/EvidPediatr/index.htm). Los autores declaran no presentar conflictos de intereses en relación con la preparación y publicación de este artículo. 
no long-term side effects during the 3-year follow-up were observed. The clinical practice guideline reviewed the literature with the aim of addressing possible therapeutic interventions in the management of children with simple febrile seizures and concluded that although there is evidence that both continuous antiepileptic therapy and intermittent therapy with oral diazepam (OD) are effective in reducing the risk of recurrence, the potential toxicities associated with antiepileptic drugs outweigh the relatively minor risk associated with simple febrile seizures. In situations of parental anxiety associated with febrile seizures, intermittent $O D$ at the onset of febrile illness may be effective in preventing recurrence.

Reviewers'commentary: continuous antiepileptic therapy or intermittent therapy with diazepam, is not recommended because the risks outweigh the benefits. In situations with severe parental anxiety associated with febrile seizures, intermittent $O D$ or RD could be used at the onset of a febrile illness. The first choice is RD at a dose of $0.33 \mathrm{mg} / \mathrm{kg}$ with a total of three doses within the first $24 \mathrm{~h}$ from the onset of fever. nosis.

Key words: Seizures, Febrile: therapy, Seizures, febrile: prevention and control, Prog-

\section{Escenario clínico}

Niña de 3 años y medio que acude a consulta tras ser atendida en el hospital por presentar un episodio de desconexión con el medio, de aproximadamente un minuto de duración, con apnea, cianosis perioral, mirada fija y flacidez del cuerpo. Posteriormente presentó relajación de esfínteres y somnolencia durante unos 20 minutos. Llevaba 24 horas con fiebre de $38{ }^{\circ} \mathrm{C}$, tos y congestión nasal. En los antecedentes personales refieren tres episodios similares, el primero a los 23 meses, siempre coincidiendo con fiebre, y que los padres achacaban a atragantamiento por mucosidad. Desarrollo psicomotor normal sin antecedentes de alteración neurológica previa. Episodios febriles frecuentes coincidiendo con infecciones de vías respiratorias altas. No antece- dentes familiares de crisis febriles ni epilepsia. Durante el ingreso se realizó EEG que fue normal. Dada de alta con diagnóstico de crisis febriles, se recomienda administrar diazepam rectal, 10 mg cada 12 horas, en caso de fiebre mayor de $38{ }^{\circ} \mathrm{C}$, durante los 2 primeros días del proceso.

Los padres están muy preocupados y preguntan si con esta medicación pueden prevenir que vuelva a ocurrirle.

Se decide revisar el tema y se les cita en unos días para informarles.

\section{Pregunta clínica}

En niños con convulsiones febriles ¿está indicado el tratamiento profiláctico con anticonvulsivantes al inicio del proceso febril para prevenir las recurrencias?

Fecha de búsqueda: enero 2009. 
Búsqueda bibliográfica

1. Biblioteca Cochrane Plus: estrategia de búsqueda "febrile seizures therapy" buscando en bases de datos de revisiones sistemáticas y en la base de datos de ensayos clínicos (CENTRAL).

2. Buscador Excelencia Clínica http: //excelenciaclinica.net con los descriptores "seizures febrile".

3. Base de datos MEDLINE, a través de PubMed: www.pubmed.org. Estrategia de búsqueda: descriptors "Seizures, Febrile/drug therapy" [Mesh] OR "Seizures, Febrile/prevention and control" [Mesh] OR "Seizures, Febrile/therapy"[Mesh]" con los limitadores: edad ("all infant:_birth-23 months, preschool child:: 2-5 years"), idioma ("English, French, Spanish") y tipo de estudio ("Meta-Analysis, Practice Guideline, Randomized Controlled Trial, Review").

En esta última base de datos se seleccionan dos artículos ${ }^{1,2}$ :

- Pavlidou E, Tzitiridou M, Panteliadis $C h$, Effectiveness of intermittent diazepam prophylaxis in febrile seizures: long-term prospective controlled study. J Child Neurol. 2006; 21:1036-40.

- Febrile seizures: clinical practice guideline for the long-term management of the child with simple febrile seizures. Pediatrics. 2008;

121:1281-6.

\section{Resumen estructurado} de los artículos seleccionados

Pavlidou E, Tzitiridou M, Panteliadis Ch. Effectiveness of intermittent diazepam prophylaxis in febrile seizures: long-term prospective controlled study. J Child Neurol. 2006;21:1036-40.

Objetivo: evaluar la eficacia de la profilaxis intermitente con diazepam rectal (DR) en la prevención de las convulsiones febriles (CF).

Diseño: estudio prospectivo aleatorizado doble ciego.

Emplazamiento: Departamento de Neurología Pediátrica de la Universidad de Aristotle, Thessaloniki, Grecia.

Población de estudio: se distribuyeron 139 niños con una primera CF para recibir profilaxis con DR o no profilaxis cuando tuviesen fiebre. Los criterios de inclusión fueron: no antecedente de convulsiones afebriles, desarrollo psicomotor normal, no tratamiento previo con anticonvulsivantes, y edad entre 6 meses y 3 años.

Intervención: se distribuyeron en dos grupos de forma aleatoria, comparables en cuanto a características demográficas 
(edad, sexo, antecedentes familiares de epilepsia, historia de CF en familiares de primer grado, asistencia a guardería, calendario vacunal completo) y clínicas (edad de comienzo, estatus convulsivo, CF compleja, temperatura rectal en el momento de la convulsión). El grupo A (68 niños) recibió profilaxis con DR los dos primeros días de un proceso febril cuando la temperatura rectal fue mayor de $38{ }^{\circ} \mathrm{C}$. La dosis administrada fue de $0,33 \mathrm{mg} / \mathrm{kg}$ cada 8 horas el primer día y cada 12 horas el segundo, hasta un máximo de $7,5 \mathrm{mg} /$ dosis y de 5 dosis en total. El grupo B (71 niños) no recibió profilaxis.

Medición de resultados: se siguieron durante 3 años y los niños fueron reevaluados hasta una edad media de 4,8 $+1,2$ años. Se estableció un perfil de riesgo de recurrencia en base a cinco factores pronósticos: edad de la primera convulsión inferior a 15 meses, historia de CF en familiares de primer grado, antecedentes de epilepsia en familiares de primer grado, CF compleja y episodios febriles frecuentes. Los niños con ninguno o un factor se clasificaron de bajo riesgo, los que tenían dos factores como riesgo intermedio y los que tenían tres o más de riesgo alto.

Resultados principales: durante los 3 años de seguimiento todos los niños del estudio tuvieron al menos seis episodios febriles. En el grupo de no profilaxis, hubo un $46 \%$ de recurrencias en los de bajo riesgo, $55 \%$ en los de riesgo intermedio y $83 \%$ en los de alto riesgo. En el grupo con profilaxis, la recurrencia fue del $33 \%$ en los de bajo riesgo, $35 \%$ en los de riesgo intermedio y $38 \%$ en los de alto riesgo. Por tanto, el uso de DR, redujo un $45 \%$ la recurrencia de $\mathrm{CF}$ en niños de alto riesgo $(p=0,005)$ y solo un $13 \%(p=0,412)$ y $20 \%(p=0,412)$ en los niños de riesgo bajo e intermedio respectivamente. Riesgo relativo $[R R]$ :

Tabla I. Recurrencia de convulsiones febriles en el grupo de alto riesgo comparando los que reciben diazepam rectal con la fiebre (grupo experimental) con los que no reciben nada (grupo control)

\begin{tabular}{l|l|l}
\hline & & IC $95 \%$ \\
\hline RA grupo control & $82,6 \%$ & $67,1 \%$ a $98,1 \%$ \\
\hline RA grupo experimental & $38,1 \%$ & $17,3 \%$ a $58,9 \%$ \\
\hline RR & 0,46 & 0,26 a 0,82 \\
\hline RRR & $-53,9 \%$ & $-74,1 \%$ a $-17,9 \%$ \\
\hline RAR & $-44,5 \%$ & $-70,4 \%$ a $-18,6 \%$ \\
\hline NNT & -3 & -6 a -2 \\
\hline
\end{tabular}

RA: riesgo absoluto; RR: riesgo relativo; RRR: reducción del riesgo relativo; RAR: reducción del riesgo absoluto; NNT: número necesario a tratar. 
0,46; intervalo de confianza del $95 \%$ [IC 95\%] 0,26-0,82. (Datos calculados por las autoras) (ver tabla I).

Conclusión de los autores: el uso intermitente de DR en los procesos febriles reduce de forma significativa el riesgo de recurrencia de CF en los niños de alto riesgo, mientras que en los de riesgo intermedio y bajo tiene una eficacia limitada. Los efectos secundarios en el grupo con DR (somnolencia, irritabilidad) fueron moderados y transitorios. No se registraron efectos secundarios a largo plazo.

Febrile seizures: clinical practice guideline for the long-term management of the child with simple febrile seizures. Pediatrics. 2008;121:1281-6.

Objetivo: analizar posibles tratamientos en niños con convulsiones febriles simples (CFS), su eficacia y los potenciales efectos adversos.

Diseño: guía de práctica clínica.

Fuentes de datos: publicaciones basadas en la evidencia desde 1998 hasta 2005 que describiesen tratamientos en niños con CFS. Es una actualización de una guía de la Academia Americana de Pediatría (AAP) ${ }^{3}$ publicada en 1999.

Selección de estudios: en la revisión original $^{3}$ se incluyeron 300 artículos a los que se añadieron otros 65 cuyo con- tenido diferenciase claramente las CFS de otro tipo de convulsiones, comparasen grupos de tratamiento y control, y describiesen la adherencia al tratamiento. La descripción de los estudios seleccionados se publicará en un próximo documento. Para la clasificación de los niveles de evidencia y fuerza de las recomendaciones se utilizó el documento de la AAP: clasificación de las recomendaciones en las guías de práctica clínica.

Extracción de datos: en el documento actual no se describe. La comisión estuvo compuesta por tres neurólogos infantiles, un pediatra y un neuroepidemiólogo. La guía fue revisada por miembros expertos de diferentes secciones y consejos de la AAP así como de la Academia Americana de Neurología.

Resultados principales: beneficios y riesgos del tratamiento intermitente con antiepilépticos: la administración de diazepam oral (DO) en dos estudios disminuyó las recurrencias de CF (en el primero el riesgo de CF por persona-año disminuyó un $44 \%$ en el grupo de DO en el momento de la fiebre. En el otro estudio se encontró un $11 \%$ de recurrencias en el grupo con DO frente a un $30 \%$ en el grupo control). En aquellos niños en los que el DO no fue eficaz, se encontró que no lo habían tomado en parte por los efectos secundarios. El tra- 
tamiento de las CF de menos de $5 \mathrm{mi}$ nutos de duración con DR o con midazolam tanto intranasal como bucal fue seguro y viable, aunque el tratamiento de las mismas no se encontró que tuviese influencia a largo plazo. Los efectos adversos de ambas medicaciones incluyen letargia, somnolencia y ataxia, siendo la depresión respiratoria muy rara incluso con la administración por vía rectal. La sedación produce un potencial enmascaramiento de los síntomas de las infecciones del sistema nervioso central.

Beneficios y riesgos de tratamiento antiepiléptico continuo: la utilización de forma continuada de fenobarbital, primidona, ácido valproico y carbamazepina produce una disminución de la recurrencia de $\mathrm{CF}$, describiéndose en todos una elevada incidencia de efectos adversos.

Beneficios y riesgos del tratamiento con antitérmicos intermitentes: la administración de paracetamol o ibuprofeno de forma pautada (cada 4 horas) o cuando sube la fiebre no ha demostrado que reduzca el riesgo de CFS. Aunque son antitérmicos seguros y eficaces, su sobredosificación puede producir importantes efectos adversos.

Conclusión: las CFS son frecuentes y benignas en niños de 6 a 60 meses con un excelente pronóstico en general. Aunque hay evidencia de que el trata- miento continuado con antiepilépticos o intermitente con DO es eficaz para reducir las recurrencias, la posible toxicidad supera los pocos riesgos de las CFS. Si la ansiedad de la familia es alta, la utilización de DO al comienzo de la fiebre puede ser eficaz para prevenir recurrencias. Los antitérmicos no previenen las CF.

Conflicto de intereses: todos los miembros firmaron la declaración voluntaria de conflicto de intereses.

Fuente de financiación: no consta.

Comentario crítico

Justificación: las CF afectan del 2 al $5 \%$ de los niños de 6 meses a 5 años. La mayoría (70-75\%) son CFS: generalizadas, duración inferior a 15 minutos, se asocian a la fiebre y no recurren en 24 horas; siendo el resto complejas (CFC): focales, múltiples y de duración superior a 15 minutos. A excepción del alto índice de recurrencias (30-50\%), no se han identificado problemas importantes a largo plazo. A pesar de ello, provocan gran ansiedad en los padres y cuidadores, que temen que la CF pueda provocar daño cerebral o incluso muerte. La administración de DO o DR al inicio de la fiebre disminuye las recurrencias hasta en un $40 \%$ en niños con mayor riesgo de recidivas, pero su uso no está exento de riesgos (sobre todo la posibi- 
lidad de enmascarar una infección del SNC). Existen controversias sobre su utilización, con gran variabilidad en la actuación en las consultas y servicios de urgencias, por lo que parece importante una nueva revisión del tema.

Validez o rigor científico: en el estudio de Pavlidou' la asignación de los pacientes fue aleatoria, aunque la asignación según días pares o impares tiene una calidad intermedia. No se administró placebo en el grupo control y aunque refiere que fue ciego para el profesional que hizo el seguimiento, probablemente este fuera un dato fácilmente deducible. Aunque solo hubo un $4 \%$ de pérdidas se realizó análisis por intención de tratar. Los autores no informan acerca de si el tratamiento de los grupos al margen de la intervención fue igual. Es posible que en el grupo de tratamiento hayan estado más pendientes de la fiebre del niño para poder adelantarse en el inicio de la administración del $D R$, lo que habría influido a favor del grupo con profilaxis. Otro dato importante para poder comparar los resultados es que el número de episodios febriles fuese el mismo, dato que confirman los autores. Los pacientes seleccionados fueron similares a los que podemos encontrar en nuestras consultas. Hay que señalar que los autores no tienen en cuenta si la convulsión recurrente fue simple o compleja, y este dato podría tener importancia para determinar el posible beneficio de la profilaxis.

La guía ${ }^{2}$ describe con claridad los criterios de inclusión. No informa acerca de cómo se realizó la búsqueda (aunque indica que se hará en una publicación posterior). Los niveles de recomendación se asignaron según las directrices de la AAP cuyos criterios se describen en la guía. Se incluyeron diferentes preferencias por parte de los pacientes y recomendaciones al respecto. No se hace referencia a cómo se resolvió el desacuerdo entre autores. Los resultados fueron revisados por miembros de distintos comités de la AAP relacionados directa o indirectamente con el objetivo de la misma. No se hace ninguna comparación con otras guías publicadas.

Interés o pertinencia clínica: la profilaxis con DR disminuye las recurrencias de CF en pacientes con tres o más factores de riesgo, lo que coincide con otras publicaciones ${ }^{4}$. Estudios incluidos en la guía de la AAP con DO no hicieron esta subclasificación, aunque también demuestran la eficacia de la administración profiláctica del DO.

En cuanto a los efectos secundarios, la diferencia de los estudios puede estar en relación con la vía de administración y las dosis, siendo menores en los que se ad- 
ministra por vía rectal. La dosis total de DO en los dos estudios a los que hace referencia la guía de la $A A P^{8,9}$ fue 0,33$0,35 \mathrm{mg} / \mathrm{kg} /$ dosis cada 8 horas hasta 24 horas después de desaparición de la fiebre. Esta dosis fue superior a la de DR del estudio de Pavlidou que administró un total de cinco dosis de DR independientemente de la duración de la fiebre. Otro estudio con $\mathrm{DR}^{4}$ también encontró efectos secundarios leves y transitorios aunque más frecuentes que el anterior, pero las dosis fueron superiores al no ajustarse al peso. En resumen, la administración rectal podría tener menos efectos secundarios, aunque esto puede ser debido a una menor dosis total.

El objetivo de la revisión son las CFS, sin embargo la mayoría de los estudios incluyen a niños con la primera CF y solo uno, junto ${ }^{9}$ con la guía de la $A A P^{2}$, se limita a CFS'. El manejo de la CF complejas depende de cada situación y no es el objetivo de esta revisión, si bien en el caso de recurrencias frecuentes y duración prolongada las recomendaciones serían las utilizadas en la profilaxis y manejo de las CFS?.

Otros autores ${ }^{5-7}$ también recomiendan no administrar anticonvulsivantes de forma continuada para la prevención de CF. En relación con la terapia intermitente coinciden, al igual que la de la $A A P$, en que en el caso de que sea necesario administrar una medicación profiláctica esta sería el diazepam, pero mientras que las dos primeras ${ }^{5,6}$ recomiendan la administración oral, la última ${ }^{7}$ recomienda como primera opción la vía rectal con dos dosis separadas 8 horas y una tercera dosis si es necesario a las 24 horas de la primera, debido a que como el $98 \%$ de las CF se repiten en las primeras 24 horas no estaría justificado administrarlo por un periodo más prolongado.

Aplicabilidad en la práctica clínica: hay evidencia de que el tratamiento profiláctico de las CF con diazepam no influye en el desarrollo posterior de epilepsia, ni en las capacidades intelectuales o motoras a largo plazo. Su utilización se asocia a efectos secundarios que sobrepasan el bajo riesgo asociado con las CFS, por lo que no es recomendable su utilización durante los procesos febriles para prevenir las recurrencias.

Si existe gran ansiedad familiar, alto riesgo de recurrencias, crisis prolongadas o difícil acceso al sistema sanitario, la profilaxis de las CFS puede ser considerada. En caso de indicarla se debería explicar a los padres la posibilidad de efectos secundarios que podrían enmascarar una infección del sistema nervioso central. El fármaco de elección sería $\mathrm{DR}$ al tener una absorción más 
rápida (alcanza nivel terapéutico a los 10 minutos de su administración) y menos efectos secundarios. Debe administrarse al inicio del proceso febril a dosis de 0,33 mg/kg/dosis (máximo de 7,5 $\mathrm{mg} /$ dosis), se puede repetir cada 8 horas, si la fiebre persiste, durante las primeras 24 horas (el $98 \%$ de las convulsiones febriles ocurren en las primeras 24 horas del comienzo de la fiebre) hasta un máximo de tres dosis. Es dudosa su necesidad en las 24 horas siguientes, debiéndose adecuar la pauta a cada paciente en particular.

\section{Resolución del escenario clínico}

En el caso que nos ocupa, teniendo en cuenta la historia y el examen físico, consideramos que se trata de CFS recurrentes y el tratamiento preventivo con diazepam no está indicado. Explicamos a los padres con detalle las características de las CFS, la tendencia a desparecer con la edad y que no hay evidencia de que las CFS causen muerte, daño cerebral, epilepsia, retraso mental, ni dificultades en el aprendizaje. También les damos instrucciones (verbales y escritas) sobre el manejo de posibles recurrencias (mantener la calma, posición de seguridad en decúbito lateral, no forzar la apertura de la boca, observar el tipo y duración de la convulsión). Explicamos que el uso de antitérmicos, aunque no previene la convulsión, alivia el malestar del niño, y que debemos evitar su sobreutilización y la "fobia" a la fiebre. En caso de recurrencia, se les puede recomendar la utilización de DR para aquellos casos en los que la convulsión dure más de 5 minutos. Teniendo en cuenta las presentaciones de las que disponemos (5 y $10 \mathrm{mg}$ ), se recomienda una dosificación aproximada de $0,3 \mathrm{mg} / \mathrm{kg}$ (2,5 mg para niños de 6 a 12 meses, 5 mg de 1 a 4 años $y$ $7,5 \mathrm{mg}$ para mayores de 4 años).

\section{Bibliografía}

1. Pavlidou E, Tzitiridou M, Panteliadis Ch. Effectiveness of intermittent diazepam prophylaxis in febrile seizures: long-term prospective controlled study. J Child Neurol. 2006;21:1036-40.

2. Febrile seizures: clinical practice guideline for the long-term management of the child with simple febrile seizures. Pediatrics. 2008;121: 1281-6.
3. Baumann, RJ. T1 Technical Report: treatment of the child with simple febrile seizures. Pediatrics.1999;103:e86.

4. Knudsen FU. Recurrence risk after first febrile seizure and effect of short-term diazepam prophylaxis. Arch Dis Child. 1985;60:1045-9.

5. Offringa $M$, Moyer V. Evidence based management of seizures associated with fever. BMJ. 2001;323:1111-4. 
6. Fetveit A. Assessment of febrile seizures in children. Eur J Pediatr. 2008;167:17-27.

7. Capovilla G, Mastrangelo M, Romeo A, Vigevano $F$. Recommendations for the management of "febrile seizures". Epilepsia. 2009;50:2-6.

8. Rosman NP, Colton T, Labazzo J. A controlled trial of diazepam administered during febrile illnesses to prevent recurrence of febrile seizures. N Engl J Med. 1993;329:79-84.
9. Verrotti A, Latini G, Corcia G. Intermitent oral diazepam prophylaxis in febrile convulsions: its efectiveness for febrile seizure recurrence. Eur J Pediatr Neurol. 2004;8:131-4.

10. Pavlidou E, Tzitiridou M, Kontopoulos E. Which factors determine febrile seizure recurrence? A prospective study. Brain Dev. 2008;30:7-13.

\section{खृक्ठ}

